\title{
MERCADO LABORAL, EDUCACIÓN SUPERIOR Y FORMACIÓN DOCENTE EN COSTA RICA
}

LABOR MARKET, HIGHER EDUCATION AND TEACHERS' TRAINING IN COSTA RICA

\section{Volumen 11, Número 1}

pp. 1-27

Este número se publicó el 30 de abril de 2011

Luis Carlos Morales Zúñiga

La revista está indexada en los directorios:

LATINDEX $\underline{\text { REDALYC}}$ IRESIE, $\underline{\text { CLASE}}, \underline{\text { DIALNET, DOAJ, E-REVIST@S}}$

La revista está incluida en los sitios:

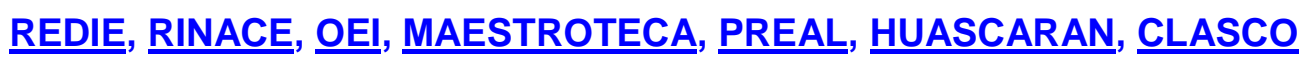




\title{
MERCADO LABORAL, EDUCACIÓN SUPERIOR Y FORMACIÓN DOCENTE EN COSTA RICA \\ LABOR MARKET, HIGHER EDUCATION AND TEACHERS' TRAINING IN COSTA RICA
}

\section{Luis Carlos Morales Zúñiga'}

\begin{abstract}
Resumen: En este ensayo se analiza la relación que existe entre la educación superior, la dinámica del mercado laboral, y la formación de docentes, en un nuevo contexto histórico-social caracterizado por la liberalización de la economía, y el avance de una concepción mercantil de la educación en Costa Rica. Se describe la relación que existe entre la desregulación de la oferta de educación superior, sobre todo privada, y la consecuente saturación de profesionales en el mercado laboral. Se analiza con especial interés la situación de los profesionales de la educación en este contexto, sobre todo, las condiciones laborales que enfrenta este sector educativo, como consecuencia de la aplicación de la lógica de mercado.
\end{abstract}

Palabras clave: EDUCACION SUPERIOR, DOCENTES, EMPLEABILIDAD, SOCIOLOGIA, MODERNIDAD

\begin{abstract}
This essay analyzes the relationship between higher education, labor market dynamics, and teacher training in a new historical and social context characterized by the liberalization of the economy, and advancing of a merchant conception of the education practice in Costa Rica. It describes the relationship between deregulation of the supply of higher education, especially private, and the resulting saturation of professional manpower in the labor market. It is analyzed with special interest, the status of the professionals of education in this context, especially the working conditions faced by this sector of the working class as a result of the application of market logic in the educational field.
\end{abstract}

Key words: HIGHER EDUCATION, TEACHERS, EMPLOYABILITY, SOCIOLOGY, MODERNITY

\footnotetext{
Magister Scientiae en Sociología, y Licenciado en la Enseñanza de los Estudios Sociales y la Educación Cívica. Profesor de la Escuela de Administración Educativa y de la Escuela de Formación Docente, en la Universidad de Costa Rica
}

Dirección electrónica: luis23m@gmail.com

Artículo recibido: 29 de setiembre, 2010

Aprobado: 7 de abril, 2011 


\section{Introducción}

En Costa Rica, la formación de docentes está en buena medida, en manos de las universidades públicas y privadas, por lo cual se podría decir que existe una suerte de descentralización en los procesos formativos formales, e institucionales de los docentes, y hay que resaltar que existen muy pocos controles que regulen la oferta de programas de formación docente. Tal forma de (des) organización y de carencia de una política nacional que regule y oriente los sistemas y procesos de formación docente (y también del resto de profesionales), genera diversas y múltiples implicaciones sobre el desarrollo de la educación en general, y por lo tanto en el desarrollo de nuestra sociedad.

Ahondar sobre el desarrollo de la educación superior, y la formación de docentes (y sus distintos correlatos, entre ellos la lógica del mercado laboral, y por ende de las condiciones laborales), en el nuevo estilo nacional de desarrollo, el cual tiene una clara orientación neoliberal, es el objetivo fundamental de este escrito. Para esto, se intenta efectuar una discusión en torno a las siguientes preguntas: ¿Cuál es la relación entre el contexto históricosocial, políticamente mediado de los últimos veinte años, y la educación superior en Costa Rica? Y ¿Qué consecuencias genera la tensionada relación entre la tendencia a la privatización de la educación superior, o la proliferación de las universidades privadas, el mercado laboral, y la formación de docentes en Costa Rica?

Reflexionar sobre estos tópicos es pertinente e imprescindible en este momento históricosocial, desde nuestro punto de vista por dos razones, las cuales justifican, nuestro planteamiento. En primera instancia, es un momento crucial en el que la relación entre la Universidad Pública, (como adalid de la educación superior en el país), y el desarrollo de la sociedad costarricense ha sido cuestionado constantemente, lo cual se refleja, sobre todo en los efectos colaterales de la discusión sobre presupuesto con que el Estado debe dotar a las universidades públicas, conocido como Fondo Especial para la Educación Superior (FEES).

Dada tal discusión entre el Consejo Nacional de Rectores (CONARE) y los representantes del Poder Ejecutivo ${ }^{2}$, sobre el establecimiento del presupuesto, se han escuchado múltiples voces que cuestionan la posición del CONARE, sin embargo, lo más

\footnotetext{
${ }^{2}$ En esta negociación interactúan, por un lado, el CONARE, compuesto por la señora Yamileth González García, rectora de la Universidad de Costa Rica (UCR), Eugenio Trejos Benavides, rector del Instituto Tecnológico Nacional (TEC), Sandra León Coto, rectora de la Universidad Nacional (UNA) y el rector de la Universidad Estatal a Distancia (UNED), Luis Guillermo Carpio Malavassi, y por otro lado ministro de educación, Leonardo Garnier Rimolo, Fernando Herrero Acosta, ministro de Hacienda, Laura Alfaro Maykall, ministra de Planificación, y Clotilde Fonseca Quesada, ministra de Ciencia y Tecnología.
} 
interesante es ver cómo el gobierno mismo, cuestiona las demandas de las Universidades Públicas (CONARE, 2010).

En segunda instancia, es frecuente escuchar opiniones de diverso origen, y leer en los periódicos nacionales, críticas a la educación costarricense, de muy variado orden. Normalmente se habla de una crisis de la educación (Molina, 2000) y en buena medida, tal crisis se relaciona entre otras cosas, con la formación de los docentes. Por ejemplo, en el segundo Informe sobre el Estado de la Educación publicado en 2008, se lee: De acuerdo con Molina (2007), en Costa Rica se observa una tendencia creciente que quienes se matriculan en las carreras de educación se encuentren educativa, cultural y socialmente menos equipados para la docencia.

A partir de los argumentos de Molina (2007), se podría inferir que, sin generalizar, las carreras de educación captan a un grupo de individuos que poseen un capital cultural menor, si se pone en relación a quienes deciden ingresar a otras carreras universitarias, posiblemente, carreras que corresponden a campos disciplinares que poseen requisitos de ingreso más rigurosos, o bien, disciplinas que poseen otro valor social/cultural, más elevado que la carrera de educación, al menos en nuestra sociedad costarricense actual.

Por otro lado, la lógica de funcionamiento del mercado laboral y del mercado de bienes culturales (servicios privados de educación superior), en relación a la proliferación de universidades privadas, y la inexistente, hasta ahora, voluntad política de parte de los grupos que han accedido al poder del Estado, por regular la relación entre formación profesional y mercado laboral, tiene algunas consecuencias sobre las cuales, la ciencia social debe pronunciarse. Entre ellas, la acentuación de la desigualdad en el acceso al capital cultural realmente válido en el mercado, la saturación y por lo tanto desvalorización de la mano de obra profesional, y la consecuente flexibilización (precarización) de las condiciones laborales, para la clase trabajadora profesional.

De modo que, en términos generales los argumentos esbozados líneas atrás, justifican los intereses de esta reflexión, sobre todo si se trata de captar y dar cuenta de una parte del fenómeno relacional que se establece entre la educación superior, la formación de docentes y el desarrollo de la sociedad costarricense. 


\section{Un nuevo contexto histórico para la educación costarricense}

El cambio en el estilo nacional de desarrollo en Costa Rica, es un proceso políticamente determinado, donde los factores políticos internos juegan un papel crucial, determinante, en el marco de algunas condiciones, dadas por factores externos, que abren un abanico de posibilidades dentro del cual una sociedad se puede mover, definirse y auto constituirse. A propósito de la noción de estilo de desarrollo, Jorge Rovira Mas, siguiendo a J. Graciarena la define de la siguiente manera:

El estilo de desarrollo constituye más bien el producto que se va decantando (a manera de valores que se difunden y de prácticas que cobran vida institucional en lo económico, en lo social, en lo político, en lo cultural y en lo medioambiental) de la interacción entre distintos proyectos políticos, estrategias de desarrollo y planteos en defensa de intereses sectoriales, los que son promovidos por elites políticas y/o por grupos económicos y sociales variados que cuentan con recursos de poder con peso diferenciado en la escena política. (Rovira Mas, 2004, p. 2)

No obstante, son aquellos proyectos políticos y estrategias de desarrollo que representan los intereses de los grupos políticamente dominante, los que logran, de manera acelerada 0 gradual, expedita o paulatina, imponerse sobre otros, no sin resistencias, o sin choques y luchas contra otros actores que poseen sus propios proyectos políticos y de desarrollo.

La preponderancia de un estilo particular de desarrollo se va imponiendo desde la esfera de lo político, (es por ello que se sostiene que los factores políticos internos son determinantes) específicamente desde el ámbito de acción del Estado, y sus instituciones. Sin embargo, esto nos plantea el problema de asumir al Estado como una entidad que toma decisiones de manera con autonomía, como si tuviese cierto nivel de conciencia y actuase de cierta manera. Ralph Milliband plantea el problema en los siguientes términos:

Hay un problema preliminar acerca del Estado al que rara vez se le presta atención, y sin embargo tiene que ser considerado para enfocar con propiedad, el examen de su naturaleza y del papel que desempeña: esto es que "el Estado" no es una cosa, no existe como tal. El término Estado designa cierto número de instituciones particulares que en su conjunto, constituyen su realidad y ejercen influencia unas en otras en calidad de partes de aquello a lo que podemos llamar sistema del Estado. (Milliband, 1985, p.50) 
Por lo tanto, para analizar el tema del cambio en el estilo nacional de desarrollo, y en el papel del Estado, y por consiguiente, una nueva forma de concebir, y desarrollar la docencia y la formación de docentes en Costa Rica, tomamos la definición de Milliband acerca del Estado como el conjunto de instituciones particulares, que constituyen en última instancia, el sistema estatal, y que se diferencia de la esfera del gobierno, el cual forma parte del Estado, pero que no es el Estado en esencia, (a pesar de que muchas veces se tienda a homologarlos), sino que ejerce en gran medida el poder del Estado. Por el acceso al poder del Estado, es decir, a constituir un gobierno, con el fin de ejercer el dominio de las instituciones que componen el aparato estatal, se lucha en la palestra electoral mediante partidos políticos.

Esta lucha por el acceso al poder, se da, en Costa Rica, en el marco de un sistema de democracia representativa, formalmente institucionalizado, con un modus procedendi legal y legítimo, donde hay competencia inter-elitaria, y derechos políticos garantizados constitucionalmente, a pesar de que la competencia en el sistema democrático sea desigual. Hay desigualdad en la esfera política como palestra del poder, lo cual Miliband, lo señala de la siguiente manera:

La igualdad política, salvo formalmente, es imposible en las condiciones del capitalismo avanzado. La vida económica no puede divorciarse de la vida política. El poder económico desigual, de magnitud y carácter de las sociedades capitalistas avanzadas, produce por necesidad desigualdad política más o menos grande, independientemente de los que pueda decir la constitución. De manera semejante, el contexto capitalista de la desigualdad generalizada en donde opera el Estado es lo que determina fundamentalmente sus políticas y acciones. ...el Estado en estas sociedades de clases es primordial e inevitablemente guardián y protector de los intereses económicos en ellas dominantes. Su objetivo y misión reales son asegurar la continuación de su predominio, y no impedirlo. (Milliband, 1985, p. 253)

En el marco de esta desigualdad, es donde operan los grupos económica y políticamente dominantes de la sociedad para imponer su visión de desarrollo. Para la sociedad costarricense, a partir de 1980 aproximadamente, sectores que han asumido el pensamiento económico neoclásico en su versión neoliberal, son los que han tenido en sus manos el acceso al gobierno, y por lo tanto han ejercido el poder del Estado, reorientando sus funciones, y sus 
instituciones, y creando nuevas formas de regular la vida social, económica, política, y por lo tanto, modificando el estilo nacional de desarrollo, en su dirección, y su ritmo.

El cambio en el estilo nacional de desarrollo en Costa Rica, se manifiesta en la paulatina desarticulación del Estado estructurado en el periodo que va de 1949 a 1979, sus alcances y la función de sus instituciones. Esa forma de Estado fue propiciadora de un desarrollo económico calificado por Rovira (2004) como socialmente orientado, y su desarticulación fue un fenómeno que se da dentro del contexto de la liberalización de la economía costarricense. Al igual que casi todos los países latinoamericanos, Costa Rica ha enfrentado desde la crisis de la deuda externa acentuada a finales de la década de 1970 y principios de los ochentas, un proceso de neoliberalización de su estructura socioeconómica y sociopolítica. Al respecto, Luis Paulino Vargas Solís afirma que:

La estrategia de liberalización en Costa Rica surge a partir de la crisis de inicios de los ochenta, la cual hacía evidente el agotamiento del viejo modelo desarrollista. Pero como ya vimos era también una crisis de insolvencia externa a raíz de la deuda externa. De estos elementos surge una propuesta que pone en cuestionamiento el papel del Estado en la economía, y el énfasis en una política de apertura económica y desarrollo exportador (Vargas Solís, 2003, p. 14).

En este contexto, juega un papel central el Consenso de Washington, el cual constituye la expresión pragmática y programática del pensamiento neoliberal para su implementación y operacionalización en algunos de los países de la periferia capitalista, específicamente América Latina. La denominación "Consenso de Washington" fue acuñada por John Williamson, economista inglés, quien se refería bajo este nombre a la serie de políticas de ajuste estructural de las economías de los países latinoamericanos bajo la dirección de los organismos financieros internacionales como del Banco Mundial (BM), el Banco Interamericano de Desarrollo (BID), o el Fondo Monetario Internacional (FMI).

Con este plan de acción, se inicia la paulatina y gradual desarticulación de los EstadosIntervencionistas latinoamericanos, los cuales habían logrado, particularmente el Estado costarricense altos índices de desarrollo y alcance de los servicios básicos, obteniendo una cobertura casi universal de servicios como salud y educación, y un desarrollo relativo de algunos sectores estratégicos como electricidad, telecomunicaciones, agua, y algunas ramas de la industria. 
En Costa Rica, al igual que el resto de América Latina, se aplicaron los Programas de Ajuste Estructural (PAES) que consistieron en una serie de reformas de la acción del Estado, lo cual implicó la transformación de las estructuras productivas afectando directamente la esfera sociocultural del país (Korten, 1997).

Las acciones de los gobiernos de Costa Rica, a partir de 1984 se han orientado básicamente a la apertura de los mercados, suprimir subsidios y todo tipo de medidas proteccionistas, a algunos sectores de la estructura productiva y económica, y reducir la participación estatal en la producción. Además han retomado la idea de que el reacceso al desarrollo se finca en el ascenso de las exportaciones, dependiente de la liberalización de los mercados de productos de capitales, y de la atracción de inversión extranjera directa, sobre todo en materia de alta tecnología, industria, y turismo.

No obstante, este estilo de desarrollo ha generado algunas consecuencias. Del proceso de liberalización de la economía, promoción de exportaciones y atracción de inversión extranjera directa no se sigue, ni se obtiene inmediatamente un desarrollo económico y social significativo, es decir, no es posible plantear una relación directamente proporcional entre apertura de fronteras, promoción de exportaciones, generación de riqueza, y aumento del índice de desarrollo humano y social ${ }^{3}$,crecimiento en las posibilidades de acceso de la sociedad (especialmente de las clases en desventaja socioeconómica) a servicios antes garantizados por el Estado, y mucho menos distribución relativamente equitativa de la riqueza (PNUD, 2007). La abolición de las fronteras y la apertura comercial suele venir acompañada de una avalancha de importaciones destructiva de las empresas nacionales y proclive a la formación de enclaves exportadores privilegiados, y en el marco de la revolución digital, enclaves que no tienen ningún tipo de encadenamiento productivo o económico con el resto del sector productivo nacional (para Costa Rica el caso de INTEL). Por otro lado la inversión extranjera se especializa en extranjerizar los mejores recursos y empresas nacionales, restando al estado-nacional la capacidad de invertir en obra pública, y mucho menos de plantear una estrategia nacional de desarrollo.

\footnotetext{
${ }^{3}$ Ver el Atlas de Desarrollo Humano Cantonal, publicado por el Programa de las Naciones Unidas para el Desarrollo, (PNUD, 2007) donde queda claro, como la pobreza humana se ha incrementado en Costa Rica, sobre todo en algunos cantones que han experimenta la atracción de inversión extranjera directa, relacionada con el sector servicios, específicamente turístico. Es decir, los cantones turísticos del pacífico norte y central costarricense. Con lo cual queda claro que de la liberalización de la economía, no necesariamente se sigue un incremento en el desarrollo social y humano del país.
} 
Los costos sociales de estas reformas han sido sumamente altos, se pasa de un Estado de Intervencionista, en lo económico y en lo social, a un Estado que se reorienta a simplemente supervisar el mercado y donde se encuentran en progresivo aumento la desigualdad social, el desempleo, la pobreza y la exclusión social. Además se acentúa la migración campo-ciudad debido a los procesos de descampenización, y el desplazo de la actividad agropecuaria causado por el abandono del papel del Estado en tal sector, lo cual conlleva a un aumento de la tugurización en las zonas urbanas.

Sin embargo, tales fenómenos por mucho tiempo se han ocultado tras las estadísticas que confirman el crecimiento de la inversión extranjera y de la economía en general, argumentando un aumento en la generación de riqueza, pero dejando de lado la variable de la distribución de la riqueza generada, la cual es apropiada por pocas manos. (Korten, 1997).

Las políticas económico-sociales que adopta el país aproximadamente a partir de la década de 1980, bajo la guía del Banco Mundial, y el Fondo Monetario Internacional señalan que las vías para el desarrollo son esencialmente, la promoción de exportaciones, la apertura comercial, la tecnología avanzada y el turismo. De este modo, a partir de esa década, el sector servicios, sobre todo privado, adquiere un gran auge, se da la apertura de la banca, sistemas de exoneración de impuestos para empresas exportadoras, e incentivos turísticos, con el fin de impulsar a este sector.

En consecuencia, de la mano de este cambio en el estilo nacional de desarrollo, se genera naturalmente, un cambio en las actividades económicas preponderantes del país, y se pasa de lo que en términos de la Comisión de Estudios para América Latina (CEPAL) sería un modelo de desarrollo hacia adentro, a un modelo de desarrollo hacia fuera, a lo cual habría que agregarle la importancia que los grupos que acceden al poder del Estado en los últimos treinta años, le han asignado a la atracción de inversión extranjera directa y al fomento de la iniciativa privada.

Paralelamente a este cambio en el estilo nacional de desarrollo que ha experimentado el país, se han dado cambios en el desarrollo de la educación en todos sus niveles. Desde la educación inicial hasta la superior, se han desarrollado desde nuestro punto de vista algunos fenómenos muy concretos, entre los cuales citamos los siguientes tres:

1. Mayor segregación en el acceso a la educación: Existen instituciones educativas que responden a las dinámicas de la desigualdad social, pues están orientadas, por sus costos de matrícula y mensualidad, a sectores bien definidos de la estructura social. 
Aquella idea de que los hijos de la elite político-económica compartían con el resto de la población el mismo sistema educativo, ha desaparecido, junto a la desaparición de los barrios pluriclasistas (Fernández, 1992). Por tanto, la proliferación de instituciones privadas de educación, en todos sus niveles, es a la vez una causa y una resultante del aumento de la desigualdad social, lo cual evidencia el carácter dialéctico del fenómeno en cuestión. Igualmente, no podríamos suponer que la educación privada es por sí misma una educación de élite, pues tampoco habría por qué suponer que es monolítica ni homogénea, ya que dentro de este tipo de educación existe una propia lógica de estratificación y desigualdad, una heterogeneidad constitutiva y constituyente.

2. Menor financiamiento para el sistema educativo público: El recorte en el gasto público, como parte de las exigencias de los Programas de Ajuste Estructural, y del proceso de liberalización de la economía ha deteriorado entre otras cosas, la infraestructura de las instituciones educativas, (Korten, 1997) la calidad de vida de algunos individuos que conforman parte del sector docente, la cantidad de estudiantes por aula, el rendimiento académico del sistema público, (y la percepción que se tiene de tal rendimiento). De la mano de ello, se tiene que la carrera docente ha sido subvaluada en el mercado laboral, pues posee una menor remuneración relativa, y menor estatus social ${ }^{4}$ (CONARE, 2008).

3. Dejad hacer, dejad pasar: el célebre aforismo atribuido a Jean-Baptiste Colbert (1619 1983), sigue teniendo toda su vigencia, y en el ámbito tanto de la sociedad como de la educación costarricense ha mostrado una enorme aplicabilidad en muchos sentidos. Dos fenómenos concretos sirven para sustentar este argumento:

- El primero tiene que ver con la poca regulación que existe sobre la educación privada superior en general, y en particular, sobre la formación de docentes que se da en estos centros de enseñanza, como se puede observar, es un problema educativo de dos aristas. Esta poca regulación ha hecho que, de la mano de la proliferación de las universidades privadas, se encuentre también una proliferación de las carreas de educación, lo cual tiene como consecuencia directa, que no existan criterios de selección

\footnotetext{
${ }^{4}$ Ver datos del último Estado de la Educación, publicado en 2008 por el CONARE, donde se pone en evidencia la brecha salarial que existe entre los profesionales de la docencia, y el resto de profesionales de otras áreas. Volumen 11, Número 1, Año 2011, ISSN 1409-4703
} 
de los estudiantes de educación, por un lado, y por otro, la sobreoferta de mano de obra docente profesional (Molina, 2007). Todo ello es posible gracias a la premisa neoclásica que supone la primacía de la libertad de elección, de comercio y de consumo. Cada cual tiene la libertad de elegir qué estudiar, dónde estudiar, y cuánto pagar por tal servicio, tomando una concepción de la educación como mercancía. Esto ignora cualquier lógica de planificación y regulación del mercado laboral, poniendo en riesgo la estructura socioocupacional del país, a favor de los patronos, pues claramente a mayor oferta de mano de obra profesional, el precio de tal mano de obra se devalúa (CONARE, 2008).

- El segundo fenómeno tiene que ver con la contratación de docentes, paralelamente a la sobre oferta de mano de obra docente, y a las políticas de flexibilización laboral en general, las condiciones de trabajo y contratación de docentes han sufrido un deterioro enorme en los últimos años, además de la baja remuneración que perciben, en comparación con otros grupos socio-ocupacionales (CONARE, 2008), los docentes enfrenta problemas de diversa índole, entre ellos, el interinazgo como condena, el atraso en los salarios, la inseguridad laboral, nombramientos entrecortados, poca estabilidad laboral, y en muchos casos, la necesidad de desplazarse hasta zonas muy alejadas de su residencia (dejando atrás a la familia) con tal de no desestimar un nombramiento, y con la esperanza puesta en un eventual traslado. Evidentemente, los fenómenos antes descritos, se encuentran estrictamente relacionados con las políticas neoliberales desarrolladas en el marco del nuevo estilo nacional de desarrollo del país.

En el siguiente apartado, profundizamos a partir de evidencia empírica y reflexión teórica, (la cual sirve de base para la argumentación esbozada), el punto número tres, el cual tiene que ver con la oferta de educación superior privada, y su correspondiente proliferación, así como la consecuencia que esto produce, es decir, la saturación del mercado laboral, fenómeno que posee a su vez, algunos considerables efectos colaterales.

\section{Para ser alguien en la vida, hay que estudiar: educación superior privada, lógica de mercado y formación docente}

En Costa Rica, existe una sentencia popular que sugiere que la única forma de llegar a ser alguien en la vida, es a través de la educación. Esto quiere decir que, a través un proceso 
de movilidad social ascendente, mediado y posibilitado por la educación, es viable alcanzar un nivel socioeconómico que permita aumentar la capacidad de consumo, y cumplir (o sentir que se está cumpliendo) con un rol social considerado como útil por el resto de la sociedad.

Lo anterior, aunado a la idea central del pensamiento (neo) liberal, que plantea la libertad casi absoluta en materia comercial, la libertad de elegir por los servicios que se quiera adquirir, y el impulso a la iniciativa privada, (premisas fundamentales del nuevo estilo nacional de desarrollo) ha redundado en una amplia proliferación (y aceptación) de la Universidad Privada, o más bien, de la Universidad Empresa, (Molina, 2009) ${ }^{5}$, como una suerte de ampliación en el acceso a la educación superior.

Es importante resaltar, que existe una tendencia global al crecimiento de la oferta de educación superior privada, sobre todo la educación de carácter corporativo, o con fines de lucro, tendencia de la cual Costa Rica es parte (Brunner, 2010).

Al respecto, Silas Casillas (2005), para analizar el caso de México, plantea que el incremento de la oferta de la educación superior, se da para solventar las necesidades de distintos sectores de la estructura social, que no lograron tener cabida en el limitado acceso que brinda la educación superior pública. Sobre todo después de la firma de programas de Ajuste Estructural en la región, se da un paulatino desfinanciamiento de la educación pública, como parte de las políticas de reducción del gasto público. Es posible, por tanto observar una tendencia al incremento de la presencia de educación superior privada, lo cual ha llevado a algunos estudiosos de los fenómenos educativos, a hablar de la preponderancia del sector y del capital privado en la educación superior, o de la privatización de la educación universitaria (Braslavsky; Cosse, 1997).

Las formas de pensamiento dominantes dentro de las corrientes neoliberales, o los llamados think tanks del capitalismo moderno, han legitimado y sostenido la necesidad de la educación superior privada, no solo como una forma de ampliar el acceso, sino, incluso, como la mejor forma de gestionar la educación superior, pues desde esta perspectiva teórica, la iniciativa privada tiene mayor capacidad organizativa. Sobre esta temática, Tilak sostiene lo siguiente:

\footnotetext{
${ }^{5}$ En un muy acertado análisis, el historiador Iván Molina, logra diferenciar claramente lo que significa una Universidad como Institución frente a una Universidad como Empresa, de modo pues, queda claro que no son comparables, puesto que su naturaleza, sus fines, sus medios, sus objetivos y sus ideales son tan diferentes como inconmensurables.
} 
In the present neo-liberal framework, there emerges yet another strong school of thought, which argues that even if the government has the resources, it is not an efficient proposal for it to expand public higher education; instead, privatization of education is the effective method to enhance access to education, and to improve equity and quality in education, besides answering the problems of resource scarcity on the part of the governments ${ }^{6}$ (Tilak, 2009, p. 8).

En nuestro país, esta dinámica salta a la vista, pues según datos obtenidos de la página web del Sistema Nacional de Acreditación de la Educación Superior ${ }^{7}$ (SINAES), existen cinco universidades estatales, y sesenta universidades privadas, lo cual convierte a Costa Rica en uno de los países latinoamericanos con mayor densidad de universidades.

Esta lógica descrita en el párrafo anterior, presenta algunas características que al día de hoy, luego de algo más de veinte años de acelerado crecimiento de la oferta de educación superior privada, resultan casi infranqueables para nuestra sociedad. Además, habría que tener en consideración, la poca o inexistente regulación y planificación de la relación entre mercado laboral y formación superior ${ }^{8}$, lo cual tiende a agravar la situación de los graduados universitarios, pues se enfrentan a un mercado laboral en extremo complejo y competitivo.

El problema de la incoherente relación entre mercado laboral y educación superior, se deriva de la mencionada saturación en la oferta de servicios de educación superior, y constituye un fenómeno que tiene consecuencias sociales de gran impacto. Existe pues, una suerte de espejismo en torno a la formación superior, de manera que el hecho de acceder a ella no necesariamente garantiza hoy, en nuestro contexto actual, un proceso de movilidad social ascendente, a diferencia de décadas anteriores. La siguiente tabla permite aclarar un poco la relación entre titulación y oferta de plazas:

\footnotetext{
${ }^{6}$ En el presente marco neo-liberal, surge otra escuela fuerte de pensamiento que sostiene que incluso si el gobierno tiene los recursos, no es una propuesta eficaz para que se expanda la educación superior pública; en su lugar, la privatización de la educación es el método más eficaz para mejorar la acceso a la educación, y mejorar la equidad y la calidad de la educación, además de responder a los problemas de escasez de recursos por parte de la los gobiernos.(traducción propia)

7 http://www.sinaes.ac.cr/acerca sinaes/resena.htm (Con acceso al 28-08-2010).

8 En Costa Rica no existe una política de Estado específica, que regule la cantidad de profesionales que provee la educación superior, tanto pública como privada, en relación a la cantidad de profesionales que necesita el mercado laboral, y por ende la sociedad como conjunto. Cabe preguntarse entonces: ¿por qué no existe una política con tales objetivos? ¿A quién o quienes beneficia esta falta de regulación? ¿Qué discursos dan sustento a esta forma de concebir el tema laboral en Costa Rica?
} 
Tabla N. 1

Títulos otorgados y su relación con ofertas laborales en Costa Rica, 2006

\begin{tabular}{lrrrr}
\hline Área & \multicolumn{3}{c}{ Títulos otorgados } & \multicolumn{2}{c}{ Ofertas laborales } \\
\cline { 2 - 5 } & Absoluto & Relativo & Absoluto & Relativo \\
\hline Artes y Letras & 735 & 2,6 & 121 & 2,8 \\
Ciencias Básicas & 1737 & 6,0 & 416 & 9,6 \\
Ciencias Sociales & 10420 & 36,2 & 1669 & 38,4 \\
Educación & 10375 & 36,0 & 677 & 15,4 \\
Recursos Naturales & 451 & 1,6 & 123 & 2,8 \\
Ingeniería & 1888 & 6,6 & 884 & 20,3 \\
Ciencias de la Salud & 3169 & 11,0 & 362 & 8,3 \\
\hline Total & $\mathbf{2 8 7 8 1}$ & $\mathbf{1 0 0}$ & $\mathbf{4 3 4 5}$ & $\mathbf{1 0 0}$ \\
\hline
\end{tabular}

Fuente: Segundo Informe sobre el Estado de la Educación, 2008.

Tal como se puede observar en la tabla anterior, la diferencia entre los títulos otorgados y la oferta laboral es drástica. En todas las aéreas existe una enorme incoherencia, siendo ciencias sociales y educación las que presentan una mayor dificultad, pues en ciencias sociales, según estos datos, hubo para el año 2006 un total de 8751 graduados que no habrían obtenido alguna oferta laboral, mientras que en educación, ese número aumenta a 9 698 graduados. Por un lado, tal como lo sostiene el Segundo Informe sobre el Estado de la Educación, esta terrible incoherencia tiene una relación directa con el desempleo abierto:

En Costa Rica el 18,7\% de la fuerza de trabajo mayor de 25 años tiene estudios superiores (INEC, 2006); junto con México y Argentina, que no superan el 16\%, forma parte de los tres países de América Latina con mayor porcentaje de trabajadores con esta característica (CINDE, 2006). El 15,4\% de esta población posee un título universitario o parauniversitario. Por su parte, el desempleo de nuevos graduados en educación superior no ha sido objeto de estudio desde la investigación realizada por CONARE en el 2001 para las universidades públicas, en la cual se determinó que el desempleo abierto de nuevos graduados ascendía a 6,9\%, muy similar a la tasa general del país, que en julio de 2002 fue de 6,4\%.Para el 2006, el desempleo en la fuerza de trabajo con algún nivel de educación superior alcanzó el 3,5\%, mientras que entre los graduados universitarios llegó al 2,3\%; la tasa nacional se ubicó en ese mismo año en el $6 \%$ (CONARE, 2008). 
Podríamos pensar, que el creciente desempleo que enfrenta la fuerza laboral con estudios superiores tiene una estrecha relación con la proliferación de la educación privada en el país, pues claramente, el contar con sesenta universidades privadas, tiene una repercusión directa sobre la cantidad de mano de obra profesional, la cual excede la demanda del mercado laboral. ¿Cuáles son las consecuencias de esta lógica? Sin duda podrían ser de muy variado orden y en variable cantidad, no obstante saltan a la vista las siguientes:

1. El desempleo de profesionales con grado universitario antes mencionado. Se da sobre todo en las carreras que poseen una mayor saturación en un sector específico del mercado laboral. Es evidente que las áreas de ciencias sociales y de educación, (ver tabla N.1) son las que enfrentan un mayor desempleo, y contratación temporal.

2. La devaluación económica y social de algunos profesionales específicos, sobre todo los profesionales docentes, se presenta en el tanto, además de ser una profesión menos remunerada en relación a otras profesiones, socialmente no es hoy en día una profesión que ostente un estatus social alto. Esto tiene que ver con la simple lógica de mercado, pues la relación oferta - demanda, hace que el precio oscile en función de esa relación, es decir, cuando la demanda en el mercado está satisfecha, y la oferta excede a la demanda, el valor de lo ofertado desciende, lo cual ha sido una premisa fundamental tanto de la economía política como de la economía clásica (Mandel, 1987). A partir de los datos del último Informe sobre el Estado de la Educación (CONARE, 2008) es posible observar la brecha salarial entre los docentes y otros grupos profesionales, lo cual sustenta el argumento anterior en relación a la devaluación de la profesión docente. La siguiente tabla muestra esa diferencia en la retribución económica: 
Tabla N. 2

Ingresos promedio de educadores y otros profesionales asalariados, 2004 - 2006

\begin{tabular}{lcccc}
\hline Especialidad & \multicolumn{3}{c}{ Grado académico } \\
\cline { 2 - 4 } & & Bachillerato & Licenciatura & Posgrado \\
\hline $\begin{array}{l}\text { Ingreso promedio de } \\
\text { educadores (Colones) }\end{array}$ & los & 297627 & 320335 & 484128 \\
$\begin{array}{l}\text { Ingreso promedio del resto de } \\
\text { profesionales (Colones) }\end{array}$ & 400707 & 595836 & 860395 \\
Brecha Promedio (\%) & & & \\
\hline
\end{tabular}

Fuente: Segundo Informe sobre el Estado de la Educación, 2008.

Sin embargo, además del desempleo y la devaluación de la retribución económica para los docentes y otros grupos profesionales, existen otros fenómenos que son consecuencia también de la enorme oferta de servicios de educación superior en Costa Rica, y la consecuente saturación de la oferta de servicios profesionales. Estos efectos tienen quizá el mismo carácter de infamia e inequidad, uno de ellos es el problema de la flexibilización laboral, o lo que la ciencia social inglesa ha llamado empleabilidad, como parte del fenómeno que el sociólogo Ulrich Beck ha denominado sociedad del riesgo (Beck, 1998).

En términos generales, existe una cierta lógica que podríamos calificar (sin temor a parecer desmesurados) como perversa, en relación al desarrollo que ha tenido la educación superior en Costa Rica y sus resultados para algunos grupos profesionales (entre ellos los docentes), puesto que de alguna forma, valiéndose de ideas como la libertad de mercado, libertad de oferta y de demanda, libertad en el acceso y consumo de servicios educativos, combinado con el mito de la movilidad social ascendente, como destino único del individuo educado a nivel superior, obtenemos un panorama sombrío caracterizado por el desempleo que ya citamos, y por otras formas de explotación de la mano de obra, pero sobre todo, por la aceptación por parte de las clases trabajadoras, de esas condiciones. Al respecto, Pierre Bourdieu, y Loic Wacquant, señalan un punto importante de esta lógica:

Son, sobre todo, algunos términos aislados de apariencia técnica, como la flexibilidad, (o su versión británica, la empleabilidad) que, por el hecho de condensar y de poner en circulación toda una filosofía del individuo y de la organización social, están capacitados para funcionar como autenticas consignas políticas, en este caso: el menos Estado, la reducción de la cobertura social y la aceptación de la precariedad salarial 
generalizada como una fatalidad, e incluso como algo beneficioso (Bourdieu; Wacquant, 2001, p. 13).

El desarrollo de este fenómeno en Costa Rica ${ }^{9}$, ha roto (exista o no consciencia de ello) con el mito fundamental de la movilidad social ascendente, la cual es obtenida mediante la educación, lo que el mundo del sentido común había condensado en la antigua sentencia: para ser alguien en la vida, hay que estudiar, (de otro modo se es nadie), ha cambiado dramáticamente, pues hoy en nuestra sociedad, enfrentamos el fenómeno de la inseguridad laboral, sobre lo cual Ralph Fevre ha llamado la atención, indicando que algunos trabajos son inseguros, pues no solo no permiten obtener un número indefinido de empleos, sino que además no permiten obtener contratos de trabajo permanentes (Fevre, 2007).

Además, otro fenómeno producto de esta lógica neoliberal, (y mercantil de los servicios educativos) es la ilusión frustrada de muchos individuos que hacen préstamos para estudiar en la educación superior. Estos préstamos son otorgados por el Fondo Nacional de Becas (FONABE), y por la Comisión Nacional de Préstamos para educación, (CONAPE) ambas instituciones estatales, siendo CONAPE, una institución creada en 1977, en el marco del Estado de orientación socialdemócrata, interventor y benefactor, bajo la administración de Daniel Oduber Quirós (1974 - 1978), durante el periodo donde la concepción de desarrollo era socialmente orientada (Rovira, 2004).

En principio, estas instituciones tenían como objetivo primordial, facilitar las condiciones de acceso a la educación, como estrategia de desarrollo intelectual, personal y profesional de algunos sectores de nuestra sociedad. Hoy en día, si bien ese carácter sigue siendo el mismo, los préstamos que otorgan estas instituciones sirven para financiar sobre todo, el consumo de servicios de educación privada, tal como lo observamos en la siguiente tabla:

\footnotetext{
${ }^{9}$ Gran parte de esta lógica, aplicada a la sociedad costarricense, se encuentra condensada en el impopular proyecto (para algunos sectores académicos y sociales) llamado Plan Escudo, el cual propone una serie de medidas a tomar, en materia económica y laboral, que posee un claro carácter de flexibilización o empleabilidad, en detrimento de la clase trabajadora, bajo el discurso de buscar una salida a la crisis económica enfrentada por el mundo capitalista desde 2008, es decir adquiere todo el carácter de una consigna política. En este plan, bajo el lema protección social y estímulo económico frente a la crisis internacional (Arias, 2009), se detallan las medidas a tomar, las cuales afectan directamente a la fuerza laboral, y no a los dueños de los medios de producción, sea de cualquier tipo de producción.
} 
Tabla N. 3

Préstamos otorgados por CONAPE 2000 -2006

\begin{tabular}{llllllll}
\hline Sector & 2000 & 2001 & 2002 & 2003 & 2004 & 2005 & 2006 \\
\hline $\begin{array}{l}\text { Universitario } \\
\text { estatal }\end{array}$ & 389 & 418 & 503 & 488 & 450 & 381 & 485 \\
$\begin{array}{l}\text { Universitario } \\
\text { Privado }\end{array}$ & 1761 & 2357 & 2731 & 2931 & 3015 & 2894 & 2830 \\
& & & & & & & \\
Total & 2150 & 2775 & 3234 & 3419 & 3465 & 3275 & 3315 \\
\hline
\end{tabular}

Fuente: Segundo Informe sobre el Estado de la Educación, 2008.

Se puede apreciar en la tabla número tres, la mayor cantidad de préstamos que otorga CONAPE, están orientados a financiar el consumo de servicios de educación superior privada. Esto denota un poco el funcionamiento de la lógica mercantil de la educación superior privada en Costa Rica, donde los recursos estatales sirven para brindar una parte del soporte de este segmento del sector privado de servicios.

No obstante, la mayor problemática, no es necesariamente que un sector privado de la economía se financie con préstamos que otorgados por instituciones estatales a terceros. El principal problema de esta lógica, son las consecuencias que enfrentan aquellos que hacen estos préstamos, sobre todo los que se ubican en los estratos bajos y medios de la estructura social, puesto que, al terminar su carrera, deben iniciar a pagar el préstamo, en un marco de condiciones desfavorables, caracterizado por la saturación del mercado laboral, la sobreoferta de mano de obra profesional, y la flexibilización laboral. De manera que la ilusión de ser alguien en la vida se enfrenta a una serie de condicionas adversas, producto de la lógica de funcionamiento del mercado laboral, las decisiones de los grupos que dirigen el Estado, y el sistema de educación superior privada.

Por otra parte, en este marco de condiciones, la inseguridad laboral crece. El devenir del nuevo estilo nacional de desarrollo, ha producido una forma aun más perversa de esa vieja sentencia, que sostenía la necesidad de estudiar con tal de ser alguien en la vida, pues para muchos, ni siquiera estudiar es ya una forma de ser alguien en la vida o más bien, en la sociedad, pues lo que se consideraba un proceso normal, es decir, estudiar para luego obtener 
un trabajo, no es ya una certeza absoluta, concreta y sólida, sino todo lo contrario, obedece a la lógica de la sociedad en la modernidad líquida ${ }^{10}$ (Bauman, 2003).

Es evidente por tanto, el desinterés del Estado, o de los grupos que han accedido al poder del Estado, en los últimos veinte años, por articular una verdadera y planificada política nacional de educación superior. Las Universidades Empresa, cuyo fin último es el lucro, han tenido por tanto un espacio económico-social propicio para proliferar, y esto ha incrementado la oferta de mano de obra profesional, lo cual se suma a la flexibilización laboral, y la inseguridad, llevando a la sociedad costarricense, a un fenómeno que algunos sociólogos europeos como Jean Phillipe Deranty (2008) han llamado precarización de la existencia, el cual tiene que ver con la aceptación como norma y habitus, de condiciones laborales que no son capaces de satisfacer el deseo individual de estabilidad y seguridad.

Además, en el capitalismo flexible de nuestros días, se desarrolla una consecuencia interesante, la cual tiene que ver con la poca capacidad de identificación que poseen los individuos aislados, para agruparse, identificarse con una causa o con un grupo. Este es un fenómeno de enorme interés, puesto que se podría suponer que un sector específico de la sociedad, en este caso, los trabajadores de la educación, por ser tantos, podrían tener eventualmente, una enorme capacidad de movilización y de negociación frente a la desigualdad e inequidad, y frente a precarización de las condiciones laborales realizada desde los espacios Estatales de toma de decisiones políticas.

Sin embargo, durante los últimos años, no hay evidencia de alguna movilización por cambiar las condiciones laborales de los docentes (Mora, 2007), y demandar no solo reivindicaciones salariales, sino además exigir mejores condiciones que permitan trabajar por una educación de calidad, lo cual va desde el número de estudiantes por grupo, la estabilidad laboral, la regulación del mercado laboral y de la formación de docentes para evitar la competencia desigual, el problema de la condición de los interinos, la mendicación de lecciones para completar cargas laborales, y muchos otros problemas.

Esta falta de cohesión social, y de integración, es plausible que esté relacionada con la poca capacidad desarrollar identificación y sentido de pertenencia respecto del grupo laboral al

\footnotetext{
${ }^{10}$ Esto es sobre lo que Zygmunt Bauman ha llamado la atención, cuando planteó la idea de la modernidad líquida, aduciendo que las antiguas certezas, las cuales eran sólidas, concretas, hoy en la modernidad, son líquidas, fluctuantes, cambiables, inestables, y el mundo del trabajo, no escapa de esta dinámica, al contrario, es uno de los que con mayor intensidad la experimenta.
} 
cual se pertenece, es decir, la dificultad para desarrollar lo que Clude Dubar (1992) ha llamado identidad laboral.

En las condiciones actuales la identidad laboral, (lejos ya de las antiguas identidades o conciencias de clase ocuparon sobre todo a las teorías marxistas en los procesos de modernización e industrialización), no resulta tan fácilmente aprehensible o si quiera legible, sobre todo en un marco de flexibilización de las condiciones laborales. Sobre el impacto de las nuevas condiciones laborales en las características identitarias subjetivas, y el carácter personal, Richard Sennett afirma que:

Caráter são os trocos pessoais a que damos valor em nós mesmos, e pelos quais buscamos que os outros nos valorizem. Como decidimos o que tem valor duradouro em nós numa sociedade impaciente, que se concentra no momento inmediato? Como se podem buscar metas a longo prazo numa economia dedicada ao curto prazo? Como se podem manter lealtades e compromissos mútuos em instituicões que vivem se desfazendo ou sendo continuamente reprojetadas? Estas as questoes sobre o carácter impostas pelo novo capitalismo flexible. (Sennet, 1999, p. 115) ${ }^{11}$

Más adelante Sennett afirmará que las identidades laborales modernas son mucho más fluidas y dinámicas que las antiguas identidades surgidas en las sociedades industriales marcadas por la fuerte división clasista (Sennet, 1999). Evidentemente las nuevas formas de trabajo suponen nuevas construcciones de identidades laborales y personales, y como lo sugerimos líneas atrás, en un marco como el que se configura en el nuevo estilo nacional de desarrollo, ese proceso supone la construcción de identidades quizá más flexibles y superficiales que las que se configuraron en momentos históricos anteriores, donde las certezas eran mayores (trabajos estables, una pensión prácticamente segura, seguridad social).

Es decir, muy probablemente los docentes que trabajaron antes del desarrollo del Estado Neoliberal en Costa Rica, tuvieron mayor identificación laboral, y evidentemente mayor capacidad de negociación y movilización reivindicativa. Por tanto, es este un contexto signado por la complejidad de los procesos de construcción de las identidades en un marco de

\footnotetext{
${ }^{11}$ Carácter son los rasgos personales a que damos valor en nosotros mismos, y por los cuales buscamos que los otros nos valoren. Cómo decidimos lo que tiene valor duradero en nosotros, en una sociedad impaciente, que se concentra en el momento inmediato? Cómo se pueden buscar metas a largo plazo en una economía dedicada al corto plazo? Cómo se pueden mantener lealtades y compromisos en instituciones que se están deshaciendo o siendo continuamente reproyectadas? Estas son las cuestiones sobre el carácter impuestas por el nuevo capitalismo flexible. (traducción propia)
} 
flexibilización característico del nuevo capitalismo, el cual desmoviliza al sector profesional docente.

Asimismo, otro problema fundamental es el de la formación de docentes, y los criterios (si es que los hay) que garantizan la calidad de los procesos formativos para este sector de profesionales, quienes se ocupan en buena medida del desarrollo de la educación en Costa Rica.

A partir de algunos datos del último Informe sobre el Estado de la Educación (CONARE, 2008), es posible observar como en Costa Rica, los criterios de selección que poseen las universidades en relación a la admisión para las carreras de formación docentes son precarios, o inexistentes. Además de ello, del total de universidades tanto públicas como privadas que existen hoy en el país, alrededor de 51 poseen programas y carreras de formación docente, por lo tanto la profesión docente es una en las cuales hay mayor cantidad de oferta de trabajadores.

Lo anterior explica por qué la profesión docente se encuentra devaluada en términos de remuneración económica, en términos de estatus social y cultural, y además, explica también las causas de la saturación del mercado laboral, la precarización de las condiciones laborales, y el desdén con que los empleadores privados, así como el gobierno, tratan a este sector de la clase trabajadora nacional. En la siguiente tabla se aprecian los requisitos con que deben cumplir los aspirantes a la docencia, para estudiar carreras de educación en algunas de las universidades nacionales:

Tabla N. 4

\section{Requisitos de admisión en planes vigentes en universidades seleccionadas que forman docentes,} 2007

\begin{tabular}{ll}
\hline Universidad & Requisitos de admisión \\
\hline Florencio del Castillo & Bachillerato en educación secundaria y entrevista \\
Universidad de Costa Rica & Bachillerato en educación secundaria y examen de admisión \\
$\begin{array}{l}\text { Universidad Internacional San } \\
\text { Isidro Labrador }\end{array}$ & Bachillerato en educación secundaria \\
Universidad Latina & Bachillerato en educación secundaria \\
Universidad Nacional & Bachillerato en educación secundaria, examen de admisión, y prueba \\
& específicamente diseñada para quienes estudian educación \\
Universidad Estatal a Distancia & Bachillerato en educación secundaria \\
\hline
\end{tabular}

Fuente: Segundo Informe sobre el Estado de la Educación, 2008. 
Tal como se puede observar, la única universidad que posee una prueba específica para acceder a las carreras de educación es la Universidad Nacional. El resto de universidades no cuentan con un requisito específico de ingreso a las carreras de educación, siendo el común denominador, contar al menos con el Bachillerato en educación secundaria como requisito. Además debería agregarse una práctica común en algunas universidades privadas, que consiste en admitir a estudiantes que tienen algunas materias de la educación secundaria pendientes, haciéndoles comprometerse a concluir esas materias en el transcurso de la carrera universitaria.

Esto denota la falta de criterios y de regulación de la educación superior privada, así como la libertad de mercado cultural que se ha desarrollado en Costa Rica, pues bajo el discurso de ampliar el acceso a la educación superior, se elimina cualquier regulación racional que busque algún mínimo de calidad en la formación profesional de los docentes.

Si se compara la situación de los requisitos en el ingreso a las carreras de formación docente de Costa Rica, con las de otros países, sobre todo con países europeos y asiáticos, e incluso con algunos latinoamericanos, se pone en evidencia el rezago en el que se encuentra nuestro país en torno a la selección de aquellos profesionales que se ocuparán de la educación de las nuevas generaciones. En la siguiente tabla observamos algunos de los criterios de ingreso a las carreras de formación docente en otros países:

Tabla N. 5

Tendencias internacionales en la selección de estudiantes para el ingreso a la formación docente

\begin{tabular}{ll}
\hline País & \multicolumn{1}{c}{ Requisitos de admisión } \\
\hline Corea del Sur & $\begin{array}{l}\text { Ingreso reservado a los estudiantes que obtuvieron el } 5 \% \text { más alto de la } \\
\text { cohorte en el sistema educativo }\end{array}$ \\
Finlandia & $\begin{array}{l}\text { Ingreso reservado a los estudiantes que obtuvieron el } 10 \% \text { más alto de } \\
\text { la cohorte en el sistema educativo }\end{array}$ \\
Canadá & Título universitario en cualquier otra área del conocimiento \\
Brasil & $\begin{array}{l}\text { Pruebas objetivas escritas. Evaluación de competencias genéricas y } \\
\text { específicas }\end{array}$ \\
México & $\begin{array}{l}\text { Pruebas objetivas escritas. Selección con propósito diagnostico. Se } \\
\text { evalúa razonamiento verbal y matemático }\end{array}$ \\
Cuba & $\begin{array}{l}\text { Pruebas objetivas escritas. Se evalúa: aptitudes académicas, } \\
\text { razonamiento verbal y matemático, cultura generale y áreas curriculares } \\
\text { formales. También se evalúa la vocación y otros aspectos } \\
\text { complementarios mediante entrevista laboral. }\end{array}$
\end{tabular}

Fuente: Elaboración propia con base en datos del Segundo Informe sobre el Estado de la Educación, 2008 Volumen 11, Número 1, Año 2011, ISSN 1409-4703 
Por tanto, si observamos los requerimientos que existen en otros países para estudiar carreras relacionadas con docencia, se observa como nuestro país muestra un rezago en torno a la selección de sus educadores. Los países con sistemas educativos de alto rendimiento, como Finlandia o Cuba, han comprendido que los docentes deben ser necesariamente, aquellos individuos que han demostrado la suficiente solvencia académica, intelectual y humanista, pues tendrán en sus manos, el capital humano y social que a mediano y largo plazo, podría ser el motor del desarrollo de tales sociedades.

Sin embargo, en Costa Rica, las autoridades que han estado encargadas de definir las políticas educativas nacionales, en todos los niveles del sistema educativo, desde la educación inicial hasta la superior, no han tenido la suficiente capacidad para articular, una estrategia de mejora en los procesos y criterios de selección de los estudiantes de educación. Todo lo contrario, se ha desregulado el mercado de servicios de educación superior, dejando a la libre voluntad de la oferta y la demanda, el acceso a las carreras de educación, lo cual ha deteriorado la educación, sobre todo parte de la educación pública a nivel de primaria y secundaria, y además ha deteriorado las condiciones laborales de los docentes, así como el valor socio-cultural de la profesión.

Desde una perspectiva interpretativa, podríamos plantear que el interés del Estado ha sido entre otras cosas, contar con una enorme cantidad de profesionales docentes, con tal de devaluar económicamente la profesión y su remuneración. Un ejército de mano de obra profesional barata, y manipulable, en un contexto de flexibilización laboral.

En términos de la teoría bourdesiana sobre los procesos de reproducción social presentes en todo sistema educativo, podríamos plantear que al estar la estructura y funcionamiento de algunos sistemas de enseñanza, en función de la necesidad de producir y reproducir sus condiciones de existencia, con tal de mantenerse como un espacio de reproducción cultural, no existe por tanto, interés de las clases políticas en el poder del Estado, por desarrollar una clase docente de carácter emancipador, crítico y consciente de las distintas formas de explotación y desigualdad social que se dan en nuestra sociedad. Sobre esto, Pierre Bourdieu y J.C. Passeron señalan lo siguiente:

Todo Sistema de Enseñanza requiere un grupo de agentes especializados dotados de formación homogénea, instrumentos homogeneizados y homogeneizantes para realizar un Trabajo Pedagógico y un Trabajo Escolar específico y reglamentado. El Sistema de Enseñanza impide el ejercicio del Trabajo Escolar heterogéneo mediante condiciones 
institucionales y produce una cultura escolar rutinizada. El Sistema de Enseñanza reproduce no solo arbitrariedad cultural sino que también produce reproductores de arbitrariedad cultural (Bourdieu; Passeron, 1996, p. 99).

El planteamiento de Bourdieu y Passeron, sirve como una base teórica e interpretativa que permite captar y comprender el funcionamiento de nuestro sistema educativo. Es evidente que el interés de la voluntad política dominante en nuestro contexto histórico social, no es necesariamente, construir un sistema educativo articulado desde la educación inicial hasta la educación superior, regulando la oferta privada de servicios de educación universitaria, sobre todo en función de las necesidades del mercado laboral, y que se encuentre dentro de un marco de acción definido, y coherente a los intereses del conjunto de la sociedad.

Tampoco es interés de las élites políticas, crear un cuerpo docente, no solo profesionalmente institucionalizado, es decir titulado, sino, un grupo de docentes humanistas, intelectuales, comprometidos con la mejora de las condiciones sociales de la colectividad, y con la denuncia de las formas de dominación y desigualdad social, y que además posean condiciones laborales dignas. Esta lógica de las clases políticas que han accedido al poder del Estado durante las últimas dos décadas, adquiere sentido, en el tanto, todo sistema educativo se preocupa por el reconocimiento de su legitimidad y el desconocimiento de la violencia simbólica que ejerce (Bourdieu; Passeron, 1996).

\section{Algunas consideraciones finales}

El nuevo estilo nacional de desarrollo del país, ha configurado un nuevo marco de condiciones para la educación. Este nuevo contexto se caracteriza entre otras cosas, por la aplicación de la libertad de mercado al campo educativo, y la consecuente concepción mercantilizadora de la educación en todos sus niveles.

Entre los efectos de esta concepción del fenómeno educativo, es importante resaltar la mayor segregación en el acceso a la educación, y por tanto en el acceso al capital cultural considerado como legítimo en nuestro contexto socio-histórico. Además, las dificultades en torno al financiamiento de la educación pública, y la proliferación de la educación privada en todos sus niveles. Sobre las consecuencias de esta privatización de la educación, es importante resaltar los efectos del aumento en la oferta de educación superior privada, pues sus consecuencias reoperan sobre el resto de la estructura social. 
El desarrollo de la educación superior, sobre todo privada en Costa Rica, requiere mayor regulación y mayor intervención de la esfera política estatal. De otra forma, al dejar la oferta de educación superior al libre juego del mercado, obtenemos como resultante entre otras cosas, los problemas sobre los cuales nos hemos referido a lo largo del texto. La enorme cantidad de oferta de profesionales titulados, ha incrementado el desempleo de este sector de la sociedad, además ha ocasionado la devaluación de algunos grupos profesionales específicos.

Tal situación beneficia, claramente, a los empleadores tanto privados como públicos. En estas condiciones socio-históricas, el capital y los patronos tienen la ventaja de contar con abundante capital humano calificado, que por constituirse en una enorme oferta de mano de obra profesional, se logran imponer las condiciones de los empleadores, y hay por parte de los trabajadores de la educación, poca capacidad de diálogo y negociación colectiva.

Esto ha llevado a la sociedad costarricense, a seguir una tendencia similar a la de otros países, tanto latinoamericanos como europeos, siguiendo los estilos de la modernidad, y las formas de relaciones sociales, características de este estadio del sistema capitalista. Por tanto, a partir de la evidencia empírica analizada, se denotan las condiciones creadas por el gradual avance lógica neoliberal aplicada a la educación costarricense, donde la empleabilidad o flexibilización laboral constituyen una norma, y donde la incertidumbre de las clases trabajadoras, así como la precarización de sus condiciones laborales, son vistas no solo como aceptables, sino como el orden normal de las cosas.

En este sentido, los profesionales de la educación, (y de otros campos, como el de las ciencias sociales), han sido uno de los sectores mayormente afectados. Tal como se observó, el acceso a las carreras de formación de docentes tiene muy pocos requerimientos, y la oferta de carreras relacionadas con este campo profesional es de enorme amplitud, pues prácticamente, son las universidades especializadas en algún campo, como el derecho o la medicina, o las artes, las únicas que no ofrecen carreras de educación. Como consecuencia, se ha dado un deterioro de la formación de docentes, y sobre todo de sus condiciones laborales, por ende, de sus condiciones de vida y además de la valoración que socialmente se le confiere a la profesión y a los profesionales de la docencia.

Es necesario resaltar que el foco de interés de una política de desarrollo social, no debería concentrarse en la producción irrestricta de profesionales con grados universitarios, (en efecto, sería importante darle mayor apoyo a la educación técnica), pues esto tiene a largo plazo efectos contraproducentes, sobre todo para los profesionales mismos, y por tanto para 
algunos sectores de la sociedad, los estratos medios y bajos. Estos efectos ya son visibles y constatables con la realidad socio-laboral de Costa Rica, pues tal como lo observamos, la cantidad profesionales docentes excede la capacidad que tiene el mercado laboral por ubicar a todos estos trabajadores en algún sector de la estructura socio-ocupacional, generando desempleo, o bien condiciones laborales precarias.

En el tanto no se comprenda que un educador, de cualquier nivel debe ser necesariamente un intelectual y un humanista, y en el tanto no se dignifiquen las condiciones laborales de los docentes, la educación en Costa Rica no avanzará lo suficiente como para convertirse en el motor del desarrollo nacional, y en el eje articulador de una sociedad justa, equitativa, donde se mitiguen las formas de dominación y desigualdad social.

\section{Referencias}

Arias Sánchez, Oscar. (2009). Plan Escudo. Presentación Plan de Medidas contra la Crisis Auditorio de la Fundación Omar Dengo, San José.

Bauman, Zygmunt. (2003). Modernidad líquida (2ª . ed.). Argentina: Editorial Fondo de Cultura Económica.

Beck, Ulrich. (1998). La sociedad del riesgo. Hacia una nueva modernidad. Barcelona: Editorial Paidós.

Bourdieu, Pierre; Passeron, Jean Claude. (1996). La Reproducción: elementos para una teoría del sistema educativo. México: Fontamara.

Bourdieu, Pierre; Wacquant, Loic. (2001). Las argucias de la razón imperialista. Barcelona: Editorial Paidós.

Braslavsky, Cecilia; Cosse, Gustavo. (1997). Las actuales reformas educativas en América latina: cuatro actores, tres lógicas y ocho tensiones. Programa de Promoción de la Reforma Educativa en América Latina y el Caribe.

Bruner, José Joaquín. (2010). Educación Superior: Tendencia y Desafíos. Educación Superior Privada en Chile. Boletín de Políticas 10 -PPES-. ANILLO DE CIENCIAS SOCIALES - PROGRAMA (SOC-01) DE POLÍTICAS DE EDUCACIÓN SUPERIOR, Chile: PREAL.

Consejo Nacional de Rectores (CONARE). (2010). Ministros no dicen la verdad. Universidades públicas son transparentes. Saben para qué quieren los fondos. Con acceso al 28/07/10: http://www.rectoria.ucr.ac.cr/informe/images/pdf/Comunicado.pdf 
Consejo Nacional de Rectores (CONARE). (2008). Segundo Informe sobre el Estado de la educación. San José: Programa Estado de la Nación.

Deranty, Jean-Philippe. (2008). Work and the Precarisation of Existence. European Journal of Social Theory, 11 (4). Los Angeles, London, New Delhi and Singapore

Dubar, Claude. (1992). Formes identitaires et socialization profesionelle. Revue française de sociologie, Année, 33 (4), Paris.

Fernández, Oscar. (1992). ¿Qué valores valen hoy en Costa Rica? En: Juan Manuel Villasuso (compilador), El nuevo rostro de Costa Rica. Heredia: CEDAL.

Fevre, Ralph. (2007). Employment insecurity and social theory: the power of nightmares. Work, employment and society, 21 (3), September, Los Angeles, London, New Delhi and Singapore.

Korten, Alicia. (1997). Ajuste estructural en Costa Rica: una medicina amarga. San José: Editorial DEl.

Mandel, Ernest. (1987). Introducción a la teoría económica marxista. Serie Popular Era. México: Editorial Era.

Miliband, Ralph. (1985). El Estado en la sociedad capitalista. México: Editorial siglo XXI.

Molina Guzmán, Guillermo. (2000). Crisis de la educación costarricense, un reto sin respuestas. San José, C.R.: Litografía e imprenta LIL.(falta editorial)

Molina Jiménez, Iván. (2007). Educación y sociedad en Costa Rica: de 1821 al presente (una historia no autorizada). Ponencia preparada para el Segundo Informe Estado de la Educación. San José, Programa Estado de la Nación.

Molina Jiménez, Iván. (2009). Instituciones y empresas. Artículo de Opinión, La Nación, San José, C.R.

Mora Solano, Sindy. (2007). Acciones colectivas en la sociedad costarricense 1998-2004. Diálogos Revista Electrónica de Historia, 8 (001), Universidad de Costa Rica, San José.

Programa de las Naciones Unidas para el Desarrollo PNUD. (2007). Atlas del desarrollo humano cantonal de Costa Rica 2007. San José, C.R.: PNUD; Universidad de Costa Rica.

Rovira Mas, Jorge. (2004). El nuevo estilo nacional de desarrollo de Costa Rica y el Tratado de Libre Comercio con Estados Unidos: notas para su comprensión. En: Jornadas de Reflexión. Instituto de Investigaciones Sociales, Mesa 8, San José.

Sennett, Richard. (1999). A corrosão do caráter: as consequencias pessoais do trabalho no novo capitalismo. $3^{\circ}$ Tiragem, Rio de Janeiro: Record. 
Silas Casillas, Juan Carlos. (2005). Realidades y tendencias en la educación superior privada mexicana. Perfiles educativos, 27 (109-110). México.

Tilak, Jandhyala B.G. (2009). Private sector in higher education: A few stylized facts. Social Change: 39 (1). New Delhi.

Vargas Solís, Luis Paulino. (2003). La estrategia de liberalización económica: 1980 - 2000. Cuadernos de Historia de las Instituciones, Universidad de Costa Rica. Recuperado el 2 de julio de 2010 de: http://www-rcf.usc.edu/ clark/index.html 